\title{
Epithelial mesenchymal transition and cancer stem cells in esophageal adenocarcinoma originating from Barrett's esophagus
}

\author{
YUTAKA TOMIZAWA $^{1}$, TSUNG-TEH WU ${ }^{2}$ and KENNETH K. WANG ${ }^{1}$ \\ ${ }^{1}$ Division of Gastroenterology and Hepatology; ${ }^{2}$ Department of Laboratory Medicine and Pathology, \\ Mayo Clinic, Rochester, MN, USA
}

Received December 15, 2011; Accepted February 21, 2012

DOI: $10.3892 / \mathrm{ol} .2012 .632$

\begin{abstract}
Carcinomas comprise cohesive epithelial cells linked to one another by E-cadherin-based cell-cell junctions. Epithelial mesenchymal transition (EMT) enables carcinoma cells to migrate from the original tissue and invade into stromal components. The E-cadherin promoter is frequently repressed by specific transcriptional repressors including Snail, Slug and Twist. CD133 is known to be a marker of tumor-initiating cells in human cancers. This is the first study to characterize the transcriptional factors for E-cadherin and the representative cancer stem cell marker in specimens of early esophageal adenocarcinoma (EAC) originating from Barrett's esophagus. Ten surgically treated patients were analyzed in the present study. Immunohistochemistry was performed to determine the expression of Snail, Slug, Twist and CD133, and the results were scored. Unlike previous studies of advanced stage esophageal cancers showing the overexpression of each specific transcriptional protein, the invading edges of the tumor were found to abundantly express Snail, Slug, Twist and CD133 in our cohort. Therefore, results of this study suggest that early stage cancers predominantly comprise cells with metastatic potential and this evidence adds legitimacy to the complete removal of early EAC.
\end{abstract}

\section{Introduction}

The incidence of esophageal adenocarcinoma (EAC) and Barrett's esophagus (BE) has been on the rise (1) and overall survival for EAC, particularly when it is diagnosed at an advanced stage, is poor (2). Although esophagectomy remains the mainstay of curative treatment, it is reported to be associated with a high mortality rate of $8-20 \%$ depending on the hospital volume (3). Lymph node metastasis is common even in the early stages of EAC since the esophagus characteristically receives lymphatic supply networks. Additionally, a

Correspondence to: Dr Kenneth Wang, Division of Gastroenterology and Hepatology, Mayo Clinic, 200 1st Street SW Rochester, MN 55905, USA

E-mail: wang.kenneth@mayo.edu

Key words: epithelial mesenchymal transition, cancer stem cell, esophageal adenocarcinoma, Barrett's esophagus subset of patients who have muscularis mucosae invasion have the potential for lymph node metastasis, which eventually leads to advanced EAC (4). This evidence provides a rationale for rigorous surveillance and the complete removal of early $\mathrm{EAC}$ in BE.

Epithelial malignancy, or carcinoma, comprises cohesive epithelial cells linked to one another by E-cadherin-based cellcell junctions and it is initially separated from the stroma by the basement membrane. Local invasion through the epithelial basement membrane is the first stage of metastasis, which leads to intravasation into lymph vessels. To gain access to the stromal layer, epithelial cells have to undergo a weakening of cell-cell adhesion and a series of transfers among different cell compartments. Mounting evidence points to a crucial role for epithelial mesenchymal transition (EMT) in tumor progression. EMT involves the disruption of the intercellular junctions causing dissociation from surrounding cells and the acquisition of a migratory mesenchymal-like characteristic, enabling epithelial cells to migrate away from the original tissue and to invade into stromal components (5). The loss of E-cadherin expression has been identified in gastrointestinal cancers and is reported to be associated with cancer progression and poor prognosis (6-8). The E-cadherin promoter is frequently repressed by specific transcriptional repressors including Snail, Slug and Twist, and some of these repressors are specifically recognized at the invasive front of human cancers (9).

A direct link between EMT and cancer stem cells (CSC) was recently reported. CSCs refer to a subset of tumor cells that have the ability to self-renew and continually sustain tumorigenesis. Cells undergoing EMT may be the precursors to metastatic cancer cells, possibly even metastatic CSCs (10). CD133, originally identified as a cell surface marker of primitive hematopoietic stem cells, is a marker of tumor-initiating cells in a number of human cancers (11-13).

We hypothesized that E-cadherin transcriptional factors could also be expressed at the invading edge of early EAC. If these factors were expressed, the evidence would add legitimacy to the complete removal of early EAC. At present, no studies have assessed the expression of transcriptional factors in specimens of EAC. The aim of this study was to investigate the expression of Snail, Slug, Twist and CD133 in tissue samples from early EAC patients, and assess whether the invading edges of the tumor in the submucosa were stained for each antibody to transcriptional factor proteins. 
Table I. Semi-quantitative scored analyses of percentage of stained cells.

\begin{tabular}{lcccc}
\hline & Snail & Slug & Twist & CD133 \\
\hline $\begin{array}{l}\text { Cancer cells } \\
\text { above muscularis mucosa }\end{array}$ & 4.0 & 3.8 & 3.3 & 3.2 \\
$\begin{array}{l}\text { Invading cells } \\
\text { below muscularis mucosa }\end{array}$ & 4.0 & 3.6 & 3.1 & 3.6 \\
Non-metastatic patients & & & & 3.1 \\
Metastatic patients & 4.0 & 3.8 & 3.2 & 3.2 \\
\hline
\end{tabular}

$0,0-5 \% ; 1,6-25 \% ; 2,26-50 \% ; 3,51-75 \% ; 4,76-100 \%$.

\section{Materials and methods}

Patient tissue. Patients who were referred for esophagectomy following endoscopic surveillance during the period 1997 to 2007 were identified. The patients were identified as having mucosal cancer by endoscopic ultrasonography and were assessed by a multidisciplinary team, including experienced thoracic surgeons. The patients did not receive any chemotherapy or radiotherapy as adjuvant therapy prior to or following surgery. The Institutional Review Board approved all surgical specimens used for this study. Ten surgically treated early EAC patients including seven non-metastatic patients (p-T1N0M0, $\mathrm{n}=7 ; \mathrm{p}-\mathrm{T} 1 \mathrm{~N} 1 \mathrm{M} 0, \mathrm{n}=2 ; \mathrm{p}-\mathrm{T} 2 \mathrm{~N} 1 \mathrm{M} 0, \mathrm{n}=1)$ were analyzed in this study (TNM definitions: T1, tumor invades lamina propria or submucosa; $\mathrm{T} 2$, tumor invades muscularis propria; $\mathrm{N} 1$, regional lymph node metastasis). Slides with specimens were formalinfixed and paraffin-embedded.

Pathology assessment. The pathology assessment was performed according to our protocol as previously published (14). The diagnosis of EAC was confirmed by at least two experienced gastrointestinal pathologists. In this study, all cases were reviewed again by a single study pathologist (TTW) with expertise in BE-associated neoplasia.

Immunohistochemistry. Immunohistochemistry for Snail, Slug, Twist and CD133 was performed as follows: Slides were first deparaffinized and rehydrated with xylene and ethanol, followed by a water rinse. Antigen retrieval was then performed with sodium citrate buffer $(10 \mathrm{mM}$ sodium citrate, $0.05 \%$ Tween-20, pH 6.0). Selected blocks were sliced into four sections for immunohistochemical analysis. Three sections were incubated in a primary antibody to Snail, Slug or CD133 (rabbit polyclonal antibody, Abcam, MA, USA) overnight at $4^{\circ} \mathrm{C}$, then washed with Tris-buffered saline (TBS). Enzyme-conjugated secondary antibody was applied to the slides in TBS with $1 \%$ bovine serum albumin (BSA), and incubated for $1 \mathrm{~h}$ at room temperature. The slides were then rinsed three times with TBS. The other section was washed and incubated for $1-2 \mathrm{~h}$ with primary antibody to Twist (Twist2 antibody, mouse monoclonal, Novus Biological, Littleton, CO, USA) in 1\% BSA-phosphate-buffered saline (PBS) at room temperature, washed with TBS and applied with secondary antibody to the slide in $1 \%$ BSA-PBS. The slide was also rinsed three times with PBS.
Methods. The antibody distribution was visualized by a polarized light microscopy (Axio Scope.A1, Carl Zeiss, USA) and assessed whether the invading edges of the tumor in the submucosa were stained for each antibody. We also evaluated whether there were differences in the intensity of staining between cancer cells in the mucosa and invasive cancer cells in the submucosa. The slides were scored by a method described in a previous study (15) for i) intensity of staining $(0$, negative; 1 , weak; 2 , moderate and 3 , intense), ii) percentage of epithelial cells staining $(0,0-5 \% ; 1,6-25 \% ; 2,26-50 \% ; 3,51-75 \%$ and $4,76-100 \%)$ and iii) percentage of invasive cancer cells staining (same as epithelial cells). Cell localization (nucleus, cytoplasm or cell surface) and uniformity (focal or general) were also assessed.

\section{Results}

The four proteins were expressed in cancer cells with uniform staining in both the mucosa and submucosa with Snail being more localized in the nucleus, whereas Slug, Twist and CD133 were detected exclusively in the cytoplasm in all of the EAC specimens (Fig. 1). The intensity of staining of metastatic cancer cells in the submucosa was similar to that in the mucosa. Semiquantitative scored analyses of Snail, Slug, Twist and CD133 for overall intensity were $2.5,2.8,1.9$ and 2.4, respectively. For cancer cells in the mucosa, the scores were 4.0,3.8,3.3 and 3.2, respectively, whereas for invading cells in the submucosa the scores were 4.0, 3.6, 3.1 and 3.6, respectively. The intensity of staining and the semi-quantitative score were similar between the non-metastatic and metastatic patients (Table I).

\section{Discussion}

Tumor metastasis is essential in predicting the prognosis of cancer patients and is responsible for more than $90 \%$ of all cancer mortality (16). EAC is well known for its high incidence of lymphatic dissemination even in the early stages of the disease. Moreover, almost $30 \%$ of T1b tumors have positive lymph node metastasis (17). The lamina propria in the esophagus contains lymphatic and blood vessels unlike the rest of the gastrointestinal tract. This is clinically relevant as one of the most important prognostic factors for EAC is the depth of invasion and the presence or absence of lymphovascular invasion (18). A previous report from our group revealed that the presence of metastatic lymph nodes was significantly 

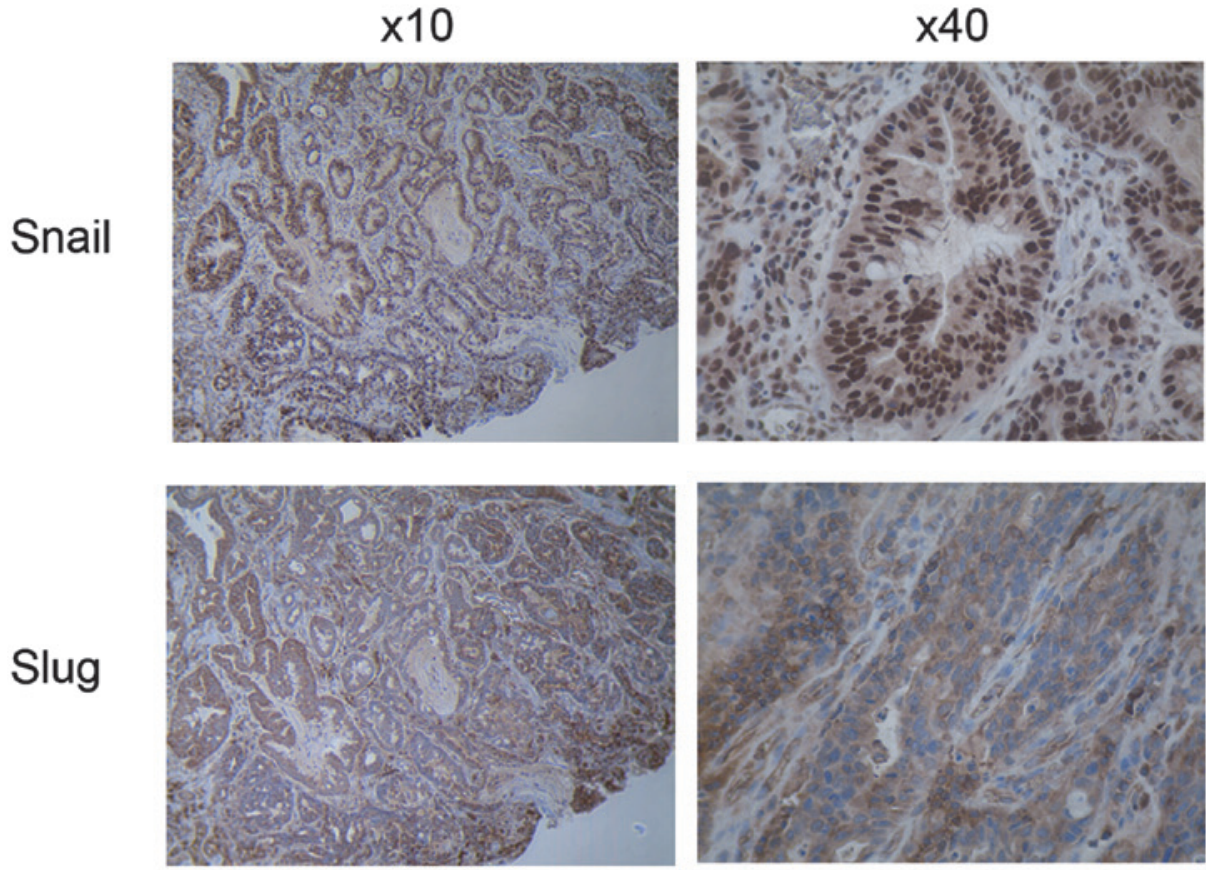

Slug
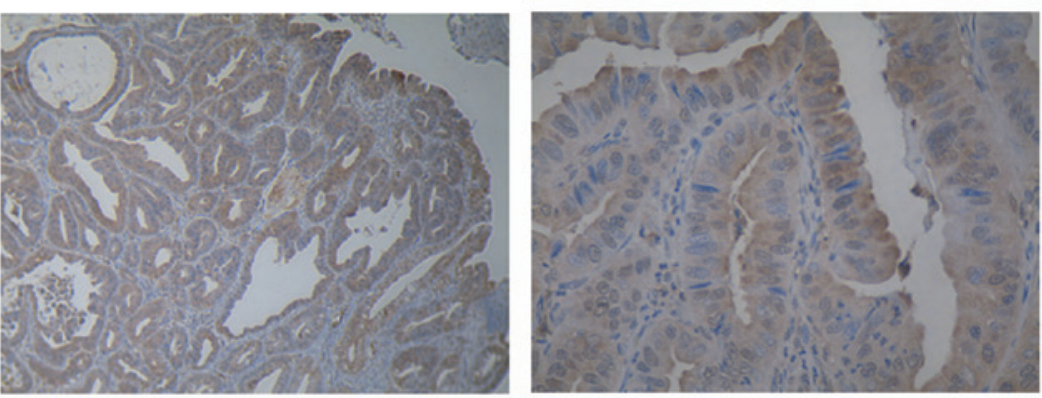

Twist
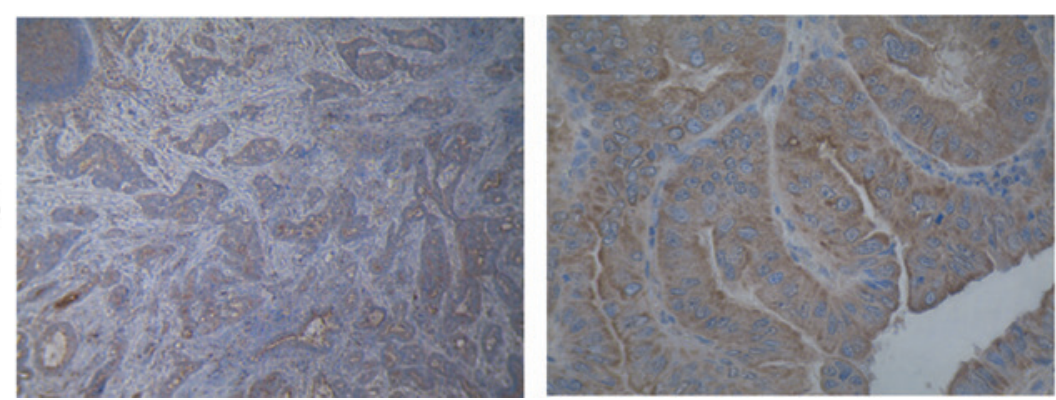

Figure 1. Immunohistochemical staining of Snail, Slug, Twist and CD133 in early esophageal adenocarcinoma.

associated with poor survival (19), and another study has shown the 5-year survival rate of patients with lymph node metastasis to be only $33 \%$ (20). EMT is a critical process during the early stage of metastasis, where cancer cells lose their epithelial characteristics and convert to a motile mesenchymal phenotype. Numerous cancer cells were demonstrated to undergo EMT during the progression towards metastatic competence. The loss of E-cadherin expression has been considered to increase tumor metastasis in gastrointestinal cancers including esophageal and gastric cancers, colorectal and hepatocellular carcinomas and pancreatic cancers (21-24). The major proteins involved in the transcriptional repression of E-cadherin are the zinc finger proteins Snail and Slug, and a basic helix-loop-helix protein Twist. Snail and Slug were shown to be capable of binding directly to E-box motifs on target gene promoters to downregulate E-cadherin expression, while Snail has been proposed to be a strong repressor of the transcription of the E-cadherin gene $(25,26)$. An increased expression of Twist has also been found to correlate with tumor invasion and metastasis (27), although the mechanism by which Twist downregulates E-cadherin expression remains to be determined. It appears that Twist is able to bind to E-box sites as a homo-dimer, but it is thought to function as a transcriptional activator in this context (28).

Following elucidation of the role of these transcriptional factors in cell culture, the expression of transcriptional factors in surgical specimens from advanced esophageal cancers was assessed. Natsugoe et al evaluated the correlation 
of Snail expression with the prognosis of 194 advanced esophageal squamous cell carcinoma (SCC) patients who underwent esophagectomy with lymph node dissection (29). In that study, tumors positive for Snail expression invaded deeper $(\mathrm{P}=0.0385)$, had more distant lymph node metastases $(\mathrm{P}=0.0051)$ and had more advanced stages $(\mathrm{P}=0.0044)$ than those that were negative for Snail expression. Patients with a reduced E-cadherin expression or positive Snail expression had poor clinical outcomes. In the preserved E-cadherin group, the overall survival rate was better in patients with a negative Snail expression than in those with a positive Snail expression $(\mathrm{P}=0.035)$. A comparison of the proportion of Snail expression in E-cadherin-preserved tumor specimens and E-cadherinreduced specimens showed no significant difference between the two groups $(\mathrm{P}=0.096)$.

The result may be explained by Snail playing a key role in EMT transition, however, Snail was not the only trigger for initiating EMT. Yuen et al studied the Twist levels in an esophagectomy specimen of SCC and examined the correlation with the occurrence of distant metastasis (30). These authors demonstrated that a high level of Twist expression was significantly associated with a greater risk of developing distant metastasis within one year of esophagectomy (OR, $3.46 ; 95 \%$ CI 1.20-9.98; $\mathrm{P}=0.022)$. They also reported that the Twist protein level was significantly increased in esophageal SCC specimens compared with non-neoplastic esophageal epithelium $(\mathrm{P}<0.001)$ and that the staining patterns observed in SCC specimens demonstrated a diffuse cytoplasmic pattern. In their study, Jethwa et al demonstrated that Snail and Slug were significantly overexpressed $(\mathrm{P}<0.05)$ in surgically treated EAC specimens compared to non-neoplastic BE specimens, and only Slug was significantly overexpressed $(\mathrm{P}<0.05)$ in EAC specimens compared to metaplastic BE specimens (15). This is the only study assessing the expression profile of the transcriptional factors in EAC specimens, and the result is comparable to those studies in advanced SCC. Although it appears that Snail, Slug and Twist have a role in EMT transition, the timeframe during which each transcriptional factor is involved in the transition has not yet been elucidated. As Jethwa et al did not identify any definite pathological stages of the specimens and used metaplastic BE specimens as a comparison to surgically treated EAC specimens, the results indicated that Slug may be involved in the late metastatic process. However, whether or not only Slug is associated with the initiation of the metastatic process in EAC remains to be determined.

Our hypothesis was that the expression of Snail, Slug and Twist in BE-related EAC was variable and might be dependent on the progression status. No studies have assessed the expression of transcriptional factors in early esophageal cancers. We selected surgically treated specimens from patients with early EAC who were initially considered for curative endoscopic therapy based on the endoscopic findings. Even in our early EAC cohort (p-T1N0M0, n=7; P-T1N1M0, n=2; p-T2N1M0, $\mathrm{n}=1$ ), Snail, Slug and Twist were all abundantly expressed in the mucosa with uniform staining. Unlike the previous studies of advanced staged esophageal cancers which demonstrated the overexpression of each specific transcriptional protein, our results indicated that early stage cancers predominantly comprise cells with metastatic potential.
The expression of $\mathrm{CD} 133$, the most representative marker of CSCs (11), was evalutated in the same cohort. Metastasis and tumor dormancy characterize numerous solid tumors and the CSC model has been proposed to account for inherent differences in tumor-regenerating capacity $(10,31,32)$. Hermann et al have found that CD133 was exclusively expressed in the tissue samples derived from pancreatic cancer patients (33). In the invasive front of pancreatic tumors, a distinct subpopulation of CD133-positive CSCs was identified and it determined the metastatic phenotype of the tumor. The EMT transition that enables potentially invasive cancer cells to disseminate from a primary tumor has also been thought to promote their selfrenewal (34). Mani et al identified a direct link between EMT and the gain of CSC properties (35). In their study, the induction of EMT in human mammary epithelial cells resulted in the expression of stem cell markers, and stem-like cells isolated from mouse or human mammary carcinomas expressed EMT markers as well. The present study is in concordance with these previous findings as we found abundant expressions of CD133 and the transcriptional factor proteins, Snail, Slug and Twist in the invading front of early, but potentially metastatic, EAC specimens. Our results emphasize the need to completely remove these early cancers.

Our findings that the markers were abundantly expressed not only partially in the actual invasive front but also in a uniform manner in the entire cancer tissue may contradict the basic theory that a subset of cancer cells within a tumor has the ability to self-renew and differentiate. This observation could be explained by a number of factors. Firstly, a higher percentage of CSCs in the fraction of cells may be identified by the antibody profile as compared with those cells that do not fit the respective profile. This may indicate that not every cell within the published profiles has CSC characteristics. There may be markers in each respective system that have yet to be identified that may help define each cancer stem cell population more precisely. Secondly, an investigation into CSCs with potential for clonal evolution in the late tumor progression stage, where more aggressive CSCs become dominant in the cancer specimen, should be conducted. Finally, it is possible that, unlike late stage cancers, early stage cancers predominantly comprise cells with metastatic potential.

In conclusion, we presented the first study to characterize the transcriptional factors for E-cadherin, Snail, Slug, Twist and the representative cancer cell marker, CD133 in the specimens of early EAC in BE. The invading edges of the tumor were found to abundantly express Snail, Slug, Twist and CD133, suggesting that early stage cancers predominantly constitute cells with metastatic potential.

\section{References}

1. Pohl H and Welch HG: The role of overdiagnosis and reclassification in the marked increase of esophageal adenocarcinoma incidence. J Natl Cancer Inst 19: 142-146, 2005.

2. Surveillance Epidemiology and End Results. http://seer.cancer. gov/csr/1975_2008/index.html. Updated November 2011.

3. Birkmeyer JD, Siewers AE, Finlayson EV, et al: Hospital volume and surgical mortality in the United States. N Engl J Med 11: 1128-1137, 2002.

4. Liu L, Hofstetter W, Rashid A, et al: Significance of the depth of tumor invasion and lymph node metastasis in superficially invasive (T1) esophageal adenocarcinoma. Am J Surg Pathol 29: 1079-1085, 2005 
5. Guarino M: Epithelial-mesenchymal transition and tumour invasion. Int J Biochem Cell Biol 39: 2153-2160, 2007.

6. Natalwala A, Spychal R and Tselepis C: Epithelial-mesenchymal transition mediated tumourigenesis in the gastrointestinal tract. World J Gastroenterol 14: 3792-3797, 2008.

7. Spaderna S, Schmalhofer O, Hlubek F, et al: A transient, EMT-linked loss of basement membranes indicates metastasis and poor survival in colorectal cancer. Gastroenterology 131: 830-840, 2006

8. Usami Y, Satake S, Nakayama F, et al: Snail-associated epithelialmesenchymal transition promotes oesophageal squamous cell carcinoma motility and progression. J Pathol 215: 330-339, 2008

9. Christofori G: New signals from the invasive front. Nature 441 444-450, 2006.

10. Visvader JE and Lindeman GJ: Cancer stem cells in solid tumors: accumulating evidence and unsolved questions. Nat Rev Cancer 8 : 755-768, 2008.

11. Mizrak D, Brittan M and Alison MR: CD133: molecule of the moment. J Pathol 214: 3-9, 2008.

12. Ricci-Vitiani L, Lombardi DG, Pilozzi E, et al: Identification and expansion of human colon-cancer-initiating cells. Nature 445: 111-115, 2007.

13. Yin $\mathrm{S}, \mathrm{Li} \mathrm{J}, \mathrm{Hu} \mathrm{C}$, et al: CD133 positive hepatocellular carcinoma cells possess high capacity for tumorigenicity. Int J Cancer 120 : 1444-1450, 2007.

14. Prasad GA, Wu TT, Wigle DA, et al: Endoscopic and surgical treatment of mucosal (T1a) esophageal adenocarcinoma in Barrett's esophagus. Gastroenterology 137: 815-823, 2009.

15. Jethwa P, Naqvi M, Hardy RG, et al: Overexpression of Slug is associated with malignant progression of esophageal adenocarcinoma. World J Gastroenterol 14: 1044-1052, 2008.

16. Hanahan D and Weinberg RA: The hallmarks of cancer Cell 100: 57-70, 2000

17. Lagarde SM, ten Kate FJ, Reitsma JB, et al: Prognostic factors in adenocarcinoma of the esophagus or gastroesophageal junction. J Clin Oncol 24: 4347-4355, 2006.

18. Hahn HP, Shahsafaei A and Odze RD: Vascular and lymphatic properties of the superficial and deep lamina propria in Barrett esophagus. Am J Surg Pathol 32: 1454-1461, 2008.

19. Badreddine RJ, Prasad GA, Lewis JT, et al: Depth of submucosal invasion does not predict lymph node metastasis and survival of patients with esophageal carcinoma. Clin Gastroenterol Hepatol 8: 248-253, 2010.

20. Westerterp M, Koppert LB, Buskens CJ, et al: Outcome of surgical treatment for early adenocarcinoma of the esophagus or gastro-esophageal junction. Virchows Arch 446: 497-504, 2005.
21. Washington K, Chiappori A, Hamilton K, et al: Expression of beta-catenin, alpha-catenin, and E-cadherin in Barrett's esophagus and esophageal adenocarcinomas. Mod Pathol 11: 805-813, 1998

22. Oda T, Kanai Y, Oyama T, et al: E-cadherin gene mutations in human gastric carcinoma cell lines. Proc Natl Acad Sci USA 91: 1858-1862, 1994.

23. Yang MH, Chen CL, Chau GY, et al: Comprehensive analysis of the independent effect of Twist and Snail in promoting metastasis of hepatocellular carcinoma. Hepatology 50: 1464-1474, 2009.

24. Lowy AM, Knight J and Groden J: Restoration of E-cadherin/ beta-catenin expression in pancreatic cancer cells inhibits growth by induction of apoptosis. Surgery 132: 141-148, 2002.

25. Cano A, Pérez-Moreno MA, Rodrigo I, et al: The transcription factor snail controls epithelial-mesenchymal transitions by repressing E-cadherin expression. Nat Cell Biol 2: 76-83, 2000.

26. Batlle E, Sancho E, Francí C, et al: The transcription factor Snail is a repressor of E-cadherin gene expression in epithelial tumour cells. Nat Cell Biol 2: 84-89, 2000.

27. Vernon AE and LaBonne C: Tumor metastasis: a new twist on epithelial - mesenchymal transitions. Curr Biol 14: 719-721, 2004.

28. Castanon I and Baylies MK: A Twist in fate: evolutionary comparison of Twist structure and function. Gene 287: 11-22, 2002 .

29. Natsugoe S, Uchikado Y, Okumura H, et al: Snail plays a key role in E-cadherin-preserved esophageal squamous cell carcinoma. Oncol Rep 17: 517-523, 2007.

30. Yuen HF, Chan YP, Wong ML, et al: Upregulation of Twist in oesophageal squamous cell carcinoma is associated with neoplastic transformation and distant metastasis. J Clin Pathol 60: 510-514, 2007.

31. Reya T, Morrison SJ, Clarke MF, et al: Stem cells, cancer, and cancer stem cells. Nature 414: 105-111, 2001.

32. Nowell PC: The clonal evolution of tumor cell populations. Science 194: 23-28, 1976.

33. Hermann PC, Huber SL, Herrler T, et al: Distinct populations of cancer stem cells determine tumor growth and metastatic activity in human pancreatic cancer. Cell Stem Cell 1: 313-323, 2007.

34. Brabletz T, Jung A, Spaderna S, et al: Opinion: migrating cancer stem cells - an integrated concept of malignant tumour progression. Nat Rev Cancer 5: 744-749, 2005.

35. Mani SA, Guo W, Liao MJ, et al: The epithelial-mesenchymal transition generates cells with properties of stem cells. Cell 133: 704-715, 2008 